\title{
APUNTES SOBRE LA INMIGRACIÓN EN LA COMUNIDAD AUTÓNOMA VALENCIANA
}

\section{Notes about immigration in theValencian Community}

\author{
Joaquín Riera Ginestar*
}

Recibido 3/04/2009; aceptado el 6/7/2010

Resumen: La Comunidad Valenciana es una región mediterránea tradicionalmente muy poblada gracias a una estructura campesina tupida derivada de unos fértiles valles litorales de regadío. No obstante, su población ha estado secularmente mal repartida, con una dicotomía entre el interior montañoso, poco poblado, y la costa, ocupada densamente. El elevado volumen de población junto con la estructura de la propiedad de la tierra (minifundismo), impulsó una corriente migratoria hacia América, Norte de África y Europa (1900-1960). La aparición desde 1959 de un marco de desarrollo industrial y de terciarización económica convierte al País Valenciano, antiguo emisor de emigrantes, en receptor de inmigración nacional (1960-1980) e internacional (desde 1995). La inmigración reciente es dual en cuanto a las motivaciones que la impulsan y que están relacionadas bien con el ocio o bien con el trabajo, dualidad que explica una distribución también diferente sobre el territorio. La nacionalidad de la población inmigrante que reside en la Comunidad Valenciana es variada, destacando la presencia británica y la rumana. La aportación de la inmigración en la Comunidad Valenciana se traduce en un impulso del decaído índice de natalidad y en un aumento del número de cotizaciones a la Seguridad Social en contexto de envejecimiento de la población nacional. La integración de la inmigración en el País Valenciano suscita dudas a través de hechos como la creciente concentración de la población inmigrante en edad escolar en centros públicos mientras se dificulta su acceso a los concertados y privados, copados por estudiantes nacionales, lo que lleva implícito un problema de segregación.

Palabras clave: Comunidad Valenciana, inmigración, educación, segregación.

Abstract: The Valencian Community has secularly been a Mediterranean region with a high rate of population, caused mainly by a solid agricultural structure of fertile irrigated valleys. However, its population has been unequally distributed over centuries, thus creating a dichotomy between the scarcely populated inland mountain area and the highly populated coastal area. This high rate of population, together with the local land property structure (i.e., small holdings) impelled between 1900-1960 a migratory stream to America, Northern Africa and Europe. From 1959, onwards, the creation of a framework for industrial development and economic tertiarization transforms the Valencian region, formerly a source of emigrants, into a a pole of attracction of both national (1960-1980) and international (since 1995) immigration. The most recent immigration is a dual phenomenon, since the reasons for emigrating are related to either leisure or work. This duality also explains the diverse settlement of the immigrants over the territory. The immigrants residing in the Valencian Community have various nationalities, although British and Romanian citizens stand out from the rest. The role of immigration in the Valencian Community becomes evident on the impulse to the previously weak birth rate and on the raise in the number Social Security affiliates in a context ageing national population. On the other hand, the integration of immigrants in the Valencian society is doubtful, as shown by facts like the increasing concentration of immigrant students in public schools while they are hindered from entering to the private education centers, which are mainly monopolizated by national students. This fact implies, therefore, a problem of segregation.

Key words: Valencian Comunity, inmigration, education, segregation.

*Licenciado en Geografía e Historia (UNED) y profesor de Enseñanza Secundaria. 


\section{INTRODUCCIÓN}

El marco físico de la Comunidad Autónoma Valenciana, y especialmente de su franja litoral, con un clima y una agricultura de regadío óptimos, ha convertido, desde la Edad Media e incluso Antigua, a las divisiones administrativas establecidas sobre esta región mediterránea en un foco de poblamiento destacado dentro de su contexto geográfico y político. No obstante, la dualidad entre montaña interior y llanura costera que caracteriza la morfología del territorio valenciano ha determinado un secular contraste demográfico entre ambas zonas, presentando el espacio montano una débil ocupación humana que en cambio es muy fuerte en la periferia litoral. A la causas físicas señaladas como raíz antigua de este desequilibrio demográfico se unen de manera determinante en los años del desarrollismo franquista factores humanos que también explican el salto cuantitativo de la población, se trata, concretamente, del triple proceso de industrialización, urbanización y terciarización que en el País Valenciano se produce en la década de los años 60 y que favorece un aumento destacable de la población (Cruz Orozco, J.; 1988).

Este hecho supone además un cambio de tendencia en los movimientos migratorios de la región valenciana, pues hasta la primera mitad del siglo XX el saldo migratorio de la región es negativo, es decir, hay una pérdida de población por la salida de emigrantes valencianos hacia el extranjero. Se da además un éxodo rural, y comienza el despoblamiento del interior a favor de la costa y, sobre todo, de Valencia. La razón de este movimiento se halla en el hecho de que, dentro de España, la Comunidad Valenciana entra muy pronto en los ciclos de la transición demográfica y con el descenso de la mortalidad el campo minifundista se siente superpoblado y comienzan los movimientos migratorios hacia las ciudades valencianas, Barcelona y Madrid (Revenga Arranz, E.; 1980). Durante la primera guerra mundial (1914-1918) hay una corriente migratoria exterior dirigida hacia a América y las huertas del sur de Francia y del norte de África (Argelia), y que es provocada, entre otras causas, porque el bloqueo comercial no permite la salida de los productos agrícolas valencianos (Menages Menages, A.; 2007). Hasta 1959 el signo de la región es, en fin, la emigración, la cual, en las comarcas del interior y en las zonas montañosas llega a ser tan importante que supera el crecimiento vegetativo, por lo que se pierde población rápidamente. Por el contrario, en las comarcas costeras y litorales, beneficiadas por la intensificación de los regadíos y por la incardinación de sus productos (cítricos) en los mercados mundiales, el crecimiento vegetativo compensa las salidas. El resultado de estos dos movimientos es un estancamiento de la población.

No obstante, al tiempo que se produce la emigración desde el campo valenciano hacia destinos nacionales y extranjeros con mayores oportunidades de trabajo entre 1900 y 1950, se observa una inmigración en Valencia procedente de las provincias limítrofes (Cuenca, Albacete y Teruel), rastreable hasta los años 20. Posteriormente, tras el retroceso demográfico y la detención momentánea de los movimientos migratorios que supone la Guerra Civil (1936-39) y después de la salida de valencianos como de otros nacionales españoles hacia Europa (Francia, Suiza, Alemania) y América (Argentina, Venezuela, Brasil) durante la posguerra (193959), desde 1960 la Comunidad Valenciana se convierte en un polo de atracción de inmigrantes, en contraste con el resto de territorios de España, que con las excepciones de las provincias vascas, Cataluña y Madrid, son emisores netos de emigrantes (Gozálvez Pérez, V.; 2004).

El triple proceso de industrialización, urbanización y terciarización en el País Valenciano, que impulsa el cambio de tendencia en los movimientos migratorios y que se concentra, como se ha señalado más arriba, en los años posteriores al Plan de Estabilización de 1959, explica que desde la década de los 60 la población valenciana entre en una fase de rápido crecimiento en la que confluyen dos factores. Por una parte, unos altos saldos vegetativos debidos al descenso de la mortalidad mientras la natalidad se mantiene aún alta; por otra, un refuerzo del flujo de inmigrantes llegados principalmente de Castilla-La Mancha, Andalucía, Extremadura y la provincia de Teruel (García Barbancho, A.;1967). Así, entre 1962 y 1975 el País Valenciano recibe 360.000 inmigrantes nacionales que llegan para trabajar en la industria y el turismo (Cruz Orozco, J.; 1988). La mayoría de ellos se radican en la provincia de Valencia, tanto en la capital como en Sagunto, y en la de Castellón. Su arraigo es claro, pues, por ejemplo, en 1975 en el área metropolitana de Valencia uno de cada tres habitantes no había nacido en la Comunidad Valenciana. Sin llegar a las cifras absolutas de Valencia, este fenómeno se repite en el valle del Vinalopó, el Campo de Alicante y en La Plana de Castellón. Sin embargo, estos son, junto con algunos puntos turísticos, los únicos del País Valenciano que reciben población. El resto de la región sufre un proceso de despoblación y éxodo rural en consonancia con el resto de España.

Por otra parte, en la fase final del franquismo, la crisis internacional de 1973 con sus secuelas de inflación, reconversión industrial y desempleo en la España de la Transición favorecen que a finales de los años 80 en el País Valenciano el saldo migratorio sea cero y que incluso llegue a ser negativo, lo que conjugado con una tasa muy débil de natalidad extensible al resto de España, deriva en un crecimiento vegetativo nulo o negativo (Gozálvez Pérez, V.; 1993). La situación se modifica radicalmente desde los últimos años de la década de los 90, con un crecimiento imparable de la población valenciana, un ascenso que es posible gracias a la inmigración extranjera. De esta manera en el último censo (2001) la Comunidad Valenciana tenía 4.162.776 habitantes, lo que da una densidad media de 179 h/km2, muy por 
encima de la media de España ( $81 \mathrm{~h} / \mathrm{km} 2)$, ganando 153.447 habitantes con respecto al censo de 1991. Este crecimiento se ha dado gracias a una tasa inmigración creciente, espectacular desde 2001, que además ha impulsado una tasa de natalidad en unos niveles muy bajos desde finales de los años ochenta.

\section{EL BOOM INMIGRATORIO DEL CAMBIO DE SIGLO}

Como se ha indicado, desde la segunda mitad de los años 90 y sobre todo desde los inicios del siglo XXI el flujo de inmigración hacia la Comunidad Valenciana se realimenta con una ingente aportación no sólo comunitaria europea (Inglaterra, Alemania, etc.) sino también extracomunitaria europea (Países del antiguo bloque comunista) y no europea (África, América y Asia). Y es que la mayor parte de los extranjeros residentes en la Comunidad Valenciana ha llegado como resultado de la fuerte inmigración concentrada en los últimos 8 años. A principios de 1999 eran 130.027 (Padrón de 1999), mientras que seis años después, a principios de 2005, eran 581.985 (Padrón de 2005). En apenas seis años se produce una entrada neta de 451.958 inmigrantes extranjeros, multiplicando por más de cuatro los niveles iniciales. De esta manera, la Comunidad Valenciana es la cuarta de España en el número de extranjeros. Si la presencia europea se debe esencialmente a las buenas condiciones climáticas (los británicos siguen siendo el principal colectivo extranjero por permisos de residencia), la inmigración laboral es básicamente el resultado del fuerte dinamismo económico que conoce esta región. En la Comunidad Valenciana encontramos una red importante y relativamente equilibrada de ciudades grandes y medias (14 poblaciones de más de 50.000 habitantes); comarcas con una agroindustria muy dinámica de citricos, hortalizas y frutales; un sector de la construcción en expansión hasta la crisis de 2008; una extraordinaria actividad turística en todo el litoral; varias zonas de industria endógena y una fuerte proporción de economía sumergida en casi todos los sectores. Estos factores conjugados con su situación demográfica y su ubicación espacial en pleno eje mediterráneo ayudan a entender el papel que la mano de obra y la inmigración tienen en esta zona.

\section{DISTRIBUCIÓN ESPACIAL DE LOS INMIGRANTES}

Un rasgo particular de la inmigración en la Comunidad Valenciana que sólo es compartido por la Comunidad Autónoma de Cataluña y en menor grado por Baleares, Murcia y Andalucía, es la presencia de dos tipos de inmigración claramente diferenciados: una basada en motivos de ocio y disfrute del tiempo libre, y otra fundamentada en causas laborales. Esta dualidad afecta de manera directa a la distribución de los inmigrantes sobre el territorio valenciano. Así, los extranjeros llegados desde los paises fundadores de la Unión Europea que disponen de ingresos para financiar su residencia y que vienen en buena medida porque han elegido la costa mediterránea como el lugar preferido para vivir durante los años que siguen a su retiro de la vida laboral, se concentran en las áreas turisticas litorales. Por su parte, la inmigración comunitaria de nuevos países miembros y la inmigración extracomunitaria, cuyos integrantes acuden buscando mejores oportunidades laborales para obtener unos ingresos que les permitan mejorar las condiciones de vida que tenían en sus países de origen, no tiene unas pautas de localización tan específicas como el primer grupo de inmigrantes. Esto es en gran parte debido a la naturaleza de los puestos de trabajo, con una cierta diversificación de actividades, pero sobre todo a la mayor dispersión espacial de los puestos de trabajo que habitualmente realizan estos inmigrantes. Es por esta dualidad en el origen de la inmigración que las principales áreas de atracción de la población extranjera en la Comunidad Valenciana, además de ser el polo tradicional originado en los años 60, esto es, las localidades turísticas litorales, se han ampliado con un crecimiento de la población inmigrante foránea en los grandes centros urbanos industriales y de servicios, en las áreas de agricultura intensiva y en los espacios rurales interiores y de agricultura extensiva, favoreciendo en este último caso la recuperación demográfica de unas zonas envejecidas y con graves pérdidas de efectivos desde la época del desarrollismo (Esparcia Pérez, J.; 2002).

El resultado final de la distribución territorial de los inmigrantes (Figura 1), según los datos del INE sobre el año 2005, es un patrón en el que los principales polos de atracción en términos absolutos son la comarca de Valencia (82.013 extranjeros), todas las comarcas del litoral alicantino (en especial El Baix Segura, con 109.923 extranjeros) y el litoral castellonense (en especial La Plana Alta, con 35.800 extranjeros). Por el contrario, las comarcas del interior (especialmente en el caso de Castellón y Valencia) se caracterizan por el escaso número de inmigrantes. Sin embargo, para valorar de forma más precisa el impacto de la inmigración conviene poner su magnitud en relación a la del conjunto de la población, ya que, como se ha dicho, también la población nacional tiende a concentrarse en algunas comarcas con mayor intensidad. Así, en cuanto al porcentaje que suponen los inmigrantes en la población total de cada comarca nos encontramos con valores que van del 4,1\% del Rincón de Ademuz al 38,9\% de La Marina Alta. Valencia, pese a ser la segunda comarca por número de extranjeros, no destaca por el peso de los mismos en su población total, ya que con un $10,3 \%$ está por debajo de la media de la Comunidad $(12,4$ $\%)$. La presencia del colectivo extranjero es particularmente destacable en algunas comarcas costeras alicantinas como la de La Marina Alta $(38,9 \%)$, El Baix Segura $(33,8 \%)$ o La Ma- 
rina Baixa $(28,9 \%)$. Otras tres comarcas, también litorales, están por encima de la media de la Comunidad: El Baix Maestrat (15,8\%), La Plana Alta (15,2\%) y La Safor (13,9\%). Por otra parte, si bien es cierto, como se ha visto, que son las comarcas litorales las que presentan generalmente un elevado porcentaje de extranjeros sobre su población total, hay comarcas interiores como La Canal de Navarrés, con un 12,3\% de población extranjera sobre el total de la población, que se acercan a las cifras de algunas zonas costeras. La consecuencia de esta diversidad dentro del patrón de predominancia de la capital valenciana y del litoral castellonense y alicantino como focos de atracción es que también a nivel provincial se observen diferencias, ya que en la provincia de Alicante los extranjeros suponen el 18,6\% de la población y en Castellón el 12,1\%, mientras que en la provincia de Valencia su peso es menor, el 8,1\%.

Figura 1. Presencia territorial a escala municipal de inmigrantes y residentes extranjeros en la Comunidad Valenciana (como \% respecto de la población total).

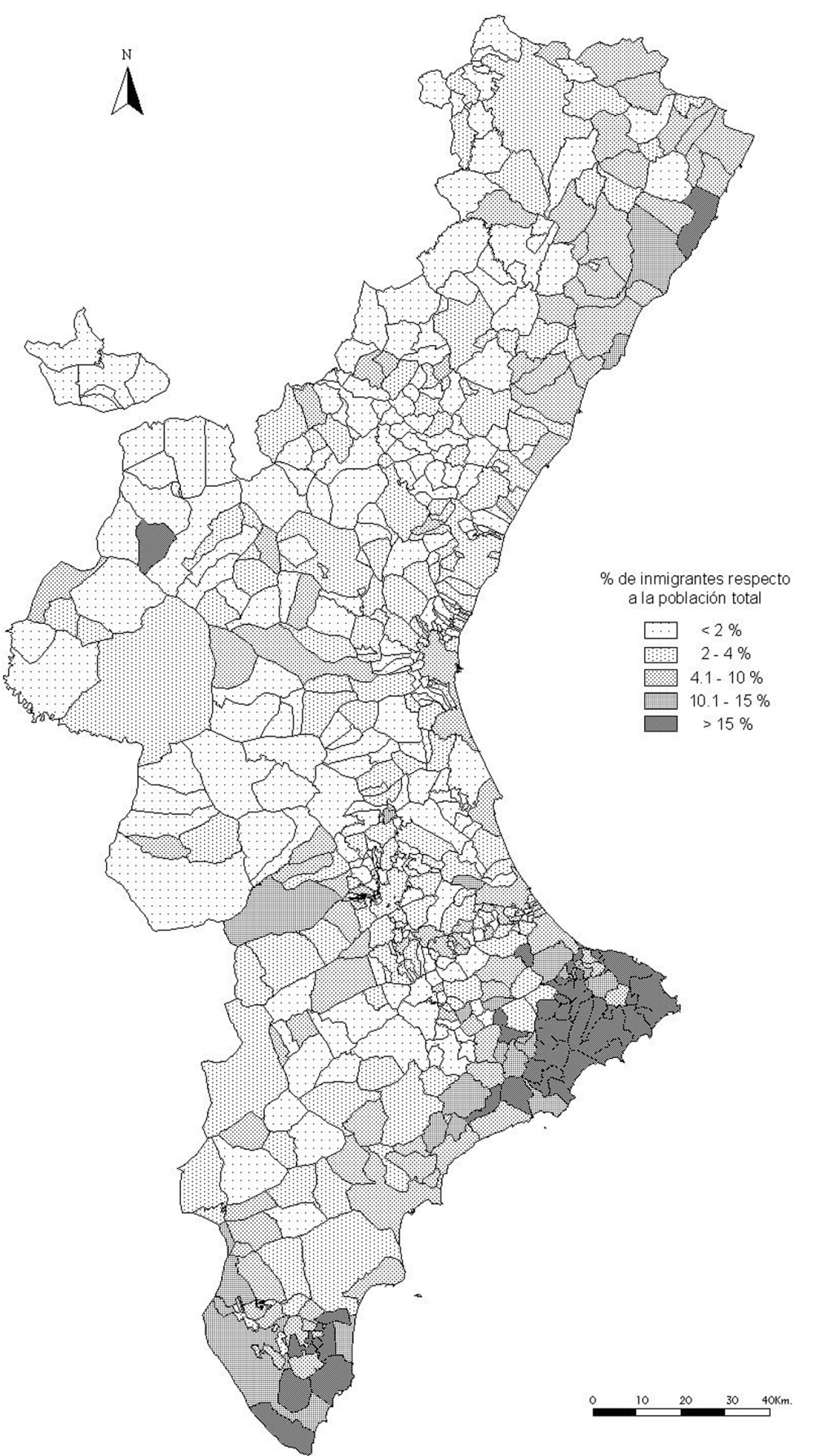

Fuente: Elaboración propia a partir del censo de Población y Vivienda (2001). INE. 


\section{PAÍSES EMISORES DE INMIGRANTES}

Por nacionalidades y a nivel global, según el padrón de 2007, Reino Unido con 121.271 efectivos es el país con más residentes en la Comunidad Valenciana, seguido a muy poca distancia por Rumania con 95.235 personas. El crecimiento de la inmigración rumana ha sido espectacular en el período 2000-2007 (Figura 2), con un aumento de 94.201 personas, por encima del incremento de la colonia europea largamente predominante, esto es, la británica que crece en el mismo lapso de tiempo en 92.458 efectivos, es decir, 1.743 personas menos que la comunidad rumana. Además, en este período de 7 años, según los datos del INE, los inmigrantes rumanos han superado a los ecuatorianos (51.425) desde 2004 y a los marroquies (56.342) desde 2005, dos grupos de inmigrantes consolidados que ocupan actualmente el cuarto y el tercer lugar, respectivamente, en el ranking de presencia extranjera en la Comunidad Valenciana (Figura 3).

Si a través de los datos del INE de 2005 se analiza en cada comarca de la Comunidad Valenciana la nacionalidad más numerosa dentro del colectivo extranjero se obtienen los siguientes resultados. El colectivo rumano es el predominante en casi todas las comarcas castellonenses (la única excepción es El Baix Maestrat) en las que supone entre el $37 \%$ y $80 \%$ de la población inmigrante. Es también el más abundante en diversas comarcas del interior de Valencia, El Camp de Morvedre, las dos Riberas y La Vall d'Albaida. Es en conjunto la primera nacionalidad en 14 comarcas. Bulgaria es la nacionalidad predominante en tres comarcas del sur de la provincia de Valencia como son La Canal de Navarrés (donde suponen el 70,4\% de los inmigrantes), La Costera $(42,3 \%)$ o La Safor $(14,8 \%)$. La inmigración marroquí es la más numerosa en El Baix Maestrat (24,7\%), L'Horta Sud (16,4\%), El Camp de Túria $(12,6 \%)$ y El Baix Vinalopó $(16,0 \%)$. Ecuador es el principal origen de los inmigrantes residentes en la comarca de València $(22,4 \%)$ y su cinturón (L'Horta Nord y L'Horta Oest, Hoya de Buñol), así como en las comarcas centrales de Alicante: L'Alt Vinalopó $(27,7 \%)$, Vinalopó Mitjà (23,3\%), L'Alcoià $(32,7 \%)$ y L'Alacanti $(11,5 \%)$. Los británicos también son nacionalidad dominante en alguna comarca, concretamente se trata de comarcas costeras y turísticas alicantinas como La Marina Alta, La Marina Baixa o el Baix Segura, además de El Comtat y El Valle de Ayora en la provincia de Valencia. Concretamente, tres comarcas representan por sí solas más del $80 \%$ de la inmigración británica. Se trata de las comarcas de El Baix Segura (38.533 personas), La Marina Alta (21.509 personas) y La Marina Baixa (10.430 personas). De hecho, los británicos son la nacionalidad más importante en esas tres comarcas con porcentajes sobre el total de extranjeros que oscilan entre en 21,7\% de la Marina Baixa y el 35,1\% de El Baix Segura.
Figura 2. Evolución de la población rumana en miles de personas en la Comunidad Valenciana. 2000-2007.

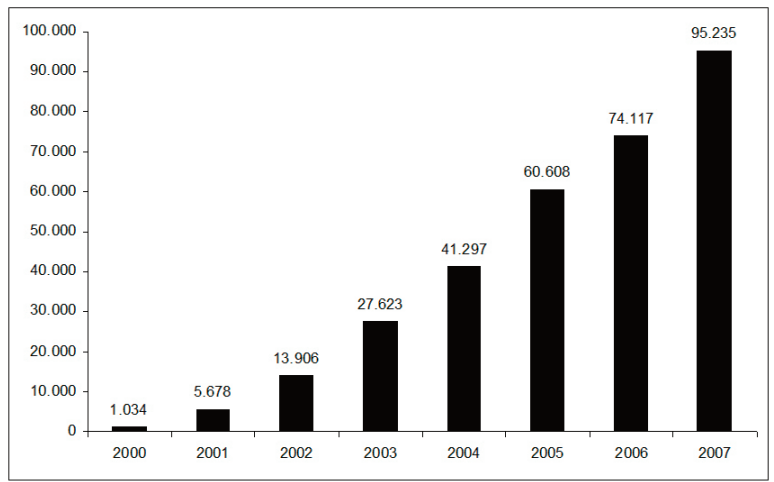

Fuente: Elaboración propia a partir de INE

Figura 3. Población extranjera en miles de personas residentes en la Comunidad Valenciana.

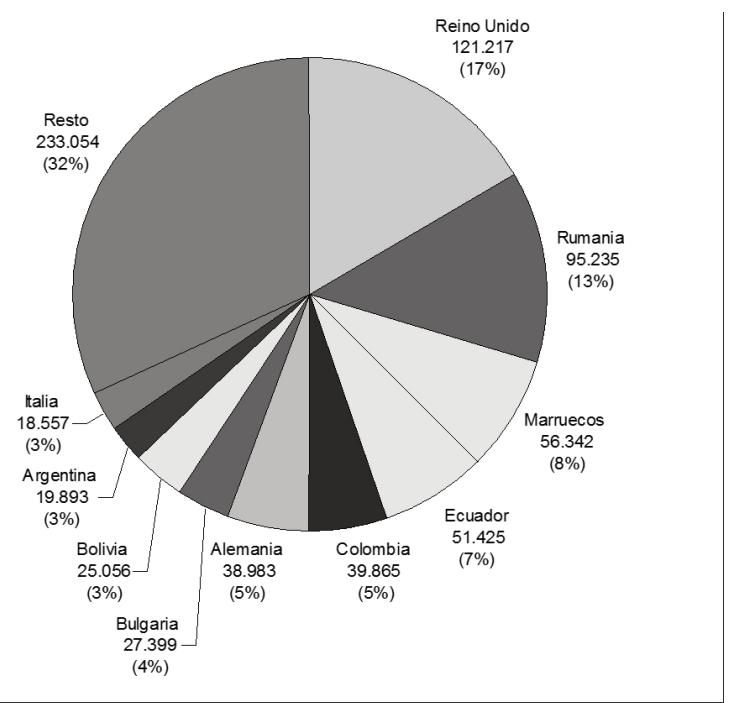

Fuente: Elaboración propia a partir del Padrón 2007. INE.

\section{NATALIDAD E INMIGRACIÓN}

La inmigración supone para la Comunidad Valenciana un aporte destacado a la natalidad. Así, siguiendo los datos proporcionados por el INE, se observa que en 2001 en la Comunidad Valenciana los nacimientos de madre española ascendieron a 39.015 mientras los nacimientos de madre extranjera fueron 3.275 , esto es, un $7,7 \%$ del total, por debajo del 8,2\% estatal. En cambio, en 2005, los nacimientos de madre española sólo ascendieron a 41.817, es decir, 2.802 más que en 2001 mientras que los nacimientos de madre extranjera se incrementaron hasta los 8.811, es decir, 5.336 más que en 2001 , lo que supone un 17,4\% del total, por encima del 15,1\% estatal o nacional. Y es que en 2005, según el INE, el Índice Sintético de Fecundidad (ISF) o indicador más usado para medir la evolución de la fecundidad en una 
población a través del número de hijos por mujer, muestra que entre las madres españolas había 1,27 hijos por mujer mientras que entre las extranjeras llegaba a 1,75 hijos por mujer.

Estos datos anteriores indican que la inmigración supone un número importante y creciente de nacimientos de niños extranjeros, aunque la aportación de éstos al rejuvenecimiento de la pirámide poblacional se lleva a cabo muy lentamente. Una parte de la aportación de la población extranjera a este rejuvenecimiento radica en compensar las elevadas defunciones de nacionales con unas tasas muy inferiores de mortalidad ${ }^{1}$ de un colectivo de extranjeros en aumento, además del suplemento que aporta la natalidad extranjera a las bajas tasas nacionales de fertilidad. En términos relativos a los nacimientos totales, según los datos de 2005, los porcentajes de madre extranjera son más elevados que la media nacional en Castellón $(22,40 \%)$ y Alicante $(20,44 \%)$, situándose Valencia $(14,19 \%)$ ligeramente por debajo de la media $(15,1 \%)$.

\section{EL MUNDO LABORAL Y LOS INMIGRANTES}

Otra aportación de la inmigración a la sociedad y la economía de la Comunidad Valenciana es la referida a las cotizaciones en la Seguridad Social. En el País Valenciano, a través de los datos de la Conselleria de Sanitat, se contabilizaba a 31 de octubre de 2007 un total de 241.607 personas extranjeras en alta laboral en la Seguridad Social, lo que significaba un 12,1\% del total de altas de la Comunidad, un 1,8 $\%$ más que en abril de 2007, y un 11,9\% de las altas estatales de extranjeros. En volumen absoluto, según los datos de Afiliados extranjeros a la Seguridad Social (MTAS) a 31 de octubre de 2007, la Comunidad Valenciana continuaba situándose en el tercer puesto entre las comunidades autónomas por afiliación extranjera, por detrás de Cataluña y Madrid, y seguida de cerca por Andalucía. Por provincias, según los datos de la Conselleria de Sanitat, la situación de la Comunidad Valenciana es muy variable. Alicante, con un 14,3\% de altas de extranjeros sobre las altas provinciales totales, y Castellón, con un 15\%, superan sensiblemente la media regional de afiliación de extranjeros (12\%); mientras que $\mathrm{Va-}$ lencia, con un 10\%, se muestra por debajo, aunque con tendencia al alza. En cualquier caso, a partir de los datos de Afiliados extranjeros a la Seguridad Social a 31 de octubre de 2007, se pone de relieve que las provincias valencianas se encuentran aún por debajo de las tasas de afiliación de trabajadores extranjeros que se producen en otras provincias como Almería (17,6\%), Lleida (16,8\%), o Murcia (16,7\%).

Estos trabajadores inmigrantes, debido a su juventud media, en torno a los 32 años (López Pino, C.; 2004), supo- nen una afiliación a la Seguridad Social que asegura un flujo mayor de cotizaciones a dicha institución a lo largo de su vida laboral, aumentando así los ingresos pero mucho menos los gastos en una época de creciente envejecimiento de la población nacional. Y esto por la mayor probabilidad que la población afiliada extranjera tiene de salir anticipadamente del sistema de la Seguridad Social, vía retorno a sus países de origen, con la minoración pertinente en las bases de cálculo de las prestaciones contributivas del sistema que ello conlleva.

Conviene señalar, por otra parte, que si bien los trabajadores inmigrantes poseen, en conjunto, niveles educativos comparables a los de los valencianos, sin embargo esa situación no se corresponde con el tipo de ocupaciones que desarrollan. En la Comunidad Valenciana, globalmente, según el Censo de Población y Viviendas de 2001 (INE), los trabajos no cualificados representaban el 16,3\%, y para los extranjeros ese porcentaje ascendía al 34,2\% correspondiendo un $13,6 \%$ al servicio doméstico y al personal de limpieza. Atendiendo al origen de los trabajadores inmigrantes, de nuevo puede distinguirse una dualidad reseñada más arriba que en este caso se traduce en que mientras que entre los extranjeros comunitarios incorporados antes de 2004 a la UE los trabajos no cualificados son poco relevantes $(1,1 \%)$, dichos trabajos son los predominantes para el resto de inmigrantes, entre los que esos porcentajes llegan a situarse por encima del 40 \% para la población extranjera de Rumanía y próxima a esa cifra en el caso de Marruecos. No obstante, algunos casos, como el de los argentinos, entre los que ese tipo de empleos no cualificados supone un porcentaje bastante más reducido, escapan a esa pauta. Otras ocupaciones importantes para los inmigrantes, junto con las reseñadas de servicio doméstico y personal de limpieza, son los servicios de restauración o los trabajos cualificados en la construcción. Por el contrario, las ocupaciones correspondientes a cuadros técnicos y directivos apenas cuentan para los extranjeros no comunitarios o comunitarios de reciente incorporación, mientras que son especialmente relevantes en el caso de los extranjeros comunitarios ingresados antes de 2004 en la UE, para los que suponen alrededor de un tercio de sus empleos totales.

\section{LA EDUCACIÓN: UN INDICADOR DE INTEGRACIÓN.}

En cuanto a la integración de los inmigrantes dentro de la sociedad de la Comunidad Valenciana hay que indicar que dicho fenómeno es más que cuestionable atendiendo a ciertas estadísticas, concretamente a los datos relacionados con la escolarización dentro de las enseñanzas no universitarias gura, Marina Alta y Marina Baja). 
de la población foránea. Primero conviene decir que en relación a las cifras de estudios terminados por la población española, según los datos la población extranjera residente y la población total en 2001, año de realización del último Censo de Población, publicado en 2003, tanto para la Comunidad Valenciana como para España, las estadísticas muestran que el nivel de los estudios terminados de la población extranjera es más elevado que el de la población nacional.

En concreto, los extranjeros en la Comunidad Valenciana son analfabetos en un 5,8\%, casi tres puntos menos que la población nacional residente en la Comunidad Valenciana; en un 9,6\% declaran no haber finalizado ningún tipo de estudios, cuando la población valenciana alcanza el 14,7\%; en un 21,4\% han terminado estudios de primer ciclo, cinco puntos menos que la población autóctona; y alcanzan un 53\% en estudios terminados de segundo grado, trece puntos más que la población valenciana censada. Tan sólo no se observan diferencias significativas en los estudios de tercer grado, situados en ambos casos en torno al $10 \%$ del total. Por otra parte, la población ocupada extranjera en la Comunidad Valenciana, según la Encuesta de Población Activa EPA del segundo trimestre de 2007 relativa a los estudios terminados de la población extranjera en el País Valenciano, muestra porcentajes más elevados que la población extranjera total en España en los estudios terminados de bachiller y ciclos formativos, tanto de grado medio como de grado superior. Correlativamente, es menor la presencia de personas analfabetas, sin estudios finalizados, con estudios primarios, con ESO, y con títulos universitarios.

Evidentemente la población extranjera residente en la Comunidad Valenciana presenta, como es lógico, grandes diferencias en sus niveles de estudios terminados según su continente y nacionalidad. Así, según el Anuario Estadístico de Inmigración de 2006 del Ministerio de Trabajo y Asuntos Sociales (MTAS), las personas procedentes de América y Oceanía muestran niveles de estudios relativamente similares a los de la población nacional, que se ven superados por los procedentes de los distintos países de Europa. Por el contrario, las personas africanas muestran una gran concentración en los niveles educativos inferiores (analfabetos, sin estudios, y estudios de primer grado), que plantean la conveniencia de una acción educativa especial para este colectivo.

Para el curso 2006-2007, el Ministerio de Educación y Ciencia (MEC) cifró en 81.932 (70.754 en el curso anterior) el número de estudiantes extranjeros de enseñanzas no universitarias en la Comunidad Valenciana, lo que suponía el $13,5 \%$ del total de estudiantes extranjeros no universitarios en España. Esto la sitúa, según la misma fuente, como la tercera comunidad por número de alumnos extranjeros, sólo precedida por Cataluña (126.027) y Madrid (124.368), y por delante de Andalucía (70.955), Murcia (28.146) o Canarias (29.674). Hay que señalar que la evolución ha sido muy dinámica, más que en el conjunto de España. En el curso 1999-
2000 la Comunidad Valenciana, con 9.461 alumnos, apenas suponía el 8,8\% del total de alumnos extranjeros. Sin embargo, si en el curso 1999-2000 la tasa de variación anual de los estudiantes extranjeros en la Comunidad Valenciana respecto del curso precedente fue incluso inferior a la de España, desde el curso 2000-2001 hasta el curso 2004-2005 esta relación se invirtió, mostrando la Comunidad Valenciana una tasa de crecimiento significativamente superior a la estatal (Figura 4). Esa tendencia se confirmó posteriormente, ya que si en el curso 2005-2006 la tasa de crecimiento en la Comunidad Valenciana (14,5\%) fue ligeramente inferior a la media española (15,3\%), en el curso 2006-2007 de nuevo la tasa de crecimiento en el País Valenciano $(15,2 \%)$ superó a la del conjunto de España (14,5\%).

Figura 4. Evolución en miles del número de estudiantes extranjeros de enseñanzas no universitarias en la Comunidad Valenciana. Cursos 1999/00-2006/07.

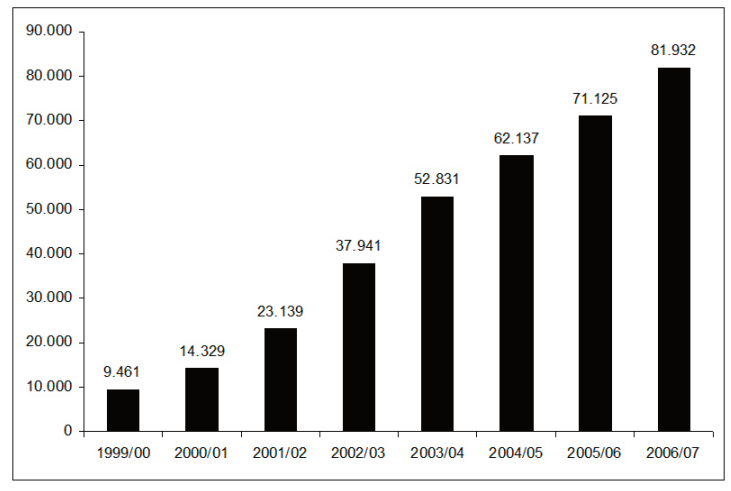

Fuente: Elaboración propia a partir de Ministerio de Educación (MEC).

En cuanto a la intensidad de la presencia del alumnado inmigrante en relación al alumnado total, la situación en el curso recogida por el MEC 2006-2007 es muy variable a escala autonómica y provincial. En la Comunidad Valenciana se observa una proporción de alumnado extranjero sobre el total superior al 10\%, aunque por debajo de Baleares, Madrid, La Rioja, Murcia y Cataluña. Ahora bien, destacan las tasas de las provincias de Alicante y Castellón, que superan el $12 \%$, muy por encima de la provincia de Valencia (8\%).

La distribución de los estudiantes extranjeros no universitarios en el curso 2005-2006 por comarcas de la Comunidad Valenciana, proporcionada por la Conselleria d'Educació, permite apreciar que en la provincia de Valencia se presenta una mayor concentración en valores absolutos en la comarca de Valencia, seguida a distancia por La Safor y I'Horta Oest. En la provincia de Castellón, destaca el volumen de población estudiantil extranjera en la Plana Alta, seguida por la Plana Baixa y el Alt Maestrat. Sin embargo, en la provincia de Alicante se observa una mayor diversificación comarcal, sobresaliendo en particular el Baix Segura, l'Alacantí, las Marinas Alta y Baixa, y el Baix Vinalopó. 
En el curso citado, según los datos de la Conselleria d'Educació, el alumnado extranjero alcanzó una media del 8,8\% respecto del alumnado total de la Comunidad Valenciana. Ahora bien, este ratio medio resultaba sensiblemente superior en comarcas como la Marina Alta (18,8\%), el Baix Segura $(16,9 \%)$, la Marina Baixa (15,4\%), el Baix Maestrat (14,3\%) y el Alto Mijares (12,0\%). En las comarcas de la provincia de Valencia la proporción de estudiantes extranjeros era algo más reducida, alcanzándose un máximo del 10,8\% en La Safor, seguido de un 9,5\% en la Canal de Navarrés.

A escala municipal, según la fuente autonómica señalada y para el mismo curso (2005-2006), en la provincia de Alicante destacaba el volumen de la población estudiantil extranjera en Alicante (4.956), Torrevieja (3.029) y Elche (2.921), seguidos por Orihuela (1.822), Benidorm (1.600), Denia (1.296) y Jávea (1.050). En la provincia de Castellón, el colectivo de estudiantes extranjeros más numeroso se daba en su capital (3.394), seguido a distancia por ciudades como Benicarló (774), Burriana (670), Villarreal (699) y Vinaroz (574). Por último, en la provincia de Valencia destacaban, además de su capital (10.265), las ciudades de Gandía (1.725), Torrent (882), Sagunto (807), Oliva (667), Mislata (616) y Ontinyent (541).

En cuanto a los países de origen de los estudiantes extranjeros en la Comunidad Valenciana durante el curso 2005-2006, según los datos del MEC, alcanzaron especial relevancia Ecuador (11.033), Colombia (7.146) y Argentina (4.506) entre los sudamericanos; Marruecos (6.586) entre los países africanos; Rumanía (7.488) y Reino Unido (6.437) entre los países europeos; $y$, a considerable distancia de los anteriores, China (1.414) como principal país asiático. El alumnado procedente de los países citados suponía en conjunto el 64,6\% del alumnado extranjero que estudiaba en la Comunidad Valenciana en el curso 2005-2006.

Por otra parte, siguiendo también los datos del MEC para el mismo curso, la distribución provincial por nacionalidades de origen mostraba algunas diferencias de interés. Así, en Castellón predominaban los estudiantes rumanos (3.551) y marroquíes (2.003), seguidos por los colombianos (766) y ecuatorianos (446). En Alicante, destacaban los procedentes de Reino Unido (5.543) y Ecuador (4.810), seguidos de Colombia (3.443) y Marruecos (2.980). Por su parte, en la provincia de Valencia sobresalía el número de estudiantes ecuatorianos (5.777), seguidos por colombianos (2.937), rumanos (2.426) y argentinos (1.813).

Pero el marcador estadístico más interesante en cuanto a la cuestión de la integración de la población inmigrante es el de la distribución del alumnado extranjero entre las redes pública y privada, particularmente desequilibrada en el País Valenciano (Figura 5), donde la red pública absorbía en el curso 2006-2007 el 86,3\% de los alumnos extranjeros, frente al $82,5 \%$ del conjunto de España (MEC). Hay que señalar, además, que dicho fenómeno aparece de forma escalonada dentro del sistema educativo, siendo leve en las primeras etapas educativas para incrementarse en las superiores, siempre dentro del marco de las enseñanzas no universitarias y obligatorias. Así, según los datos del MEC para el curso 2006-2007, en lo que respecta a la educación infantil el porcentaje de alumnos extranjeros era del 10,1\% en los centros públicos y del 2,6\% en los privados. En la educación primaria los alumnos extranjeros suponían un $17,6 \%$ en los centros públicos y un 5,5\% en los privados. En la ESO eran el $16,2 \%$ del alumnado en centros públicos y el 6,3\% en los centros privados. Como puede apreciarse, la proporción de extranjeros en los centros públicos triplica a la de los centros privados, y respecto de años anteriores se aprecia una tendencia a la ampliación de ese desfase. Esta desigualdad patente en la cobertura de la demanda educativa de la población extranjera por la oferta de las redes pública y privada, tanto concertada como no concertada, amenaza con el riesgo de que la red pública pueda sufrir, como de hecho ya está ocurriendo, un proceso grave y acumulativo de selección adversa por parte de la población autóctona y se produzca una segmentación nada deseable entre niños y jóvenes según su nacionalidad.

Figura 5. Evolución en miles del número de estudiantes extranjeros de enseñanzas no universitarias en la Comunidad Valenciana en centros públicos y privados. Cursos 2001/022006/07.

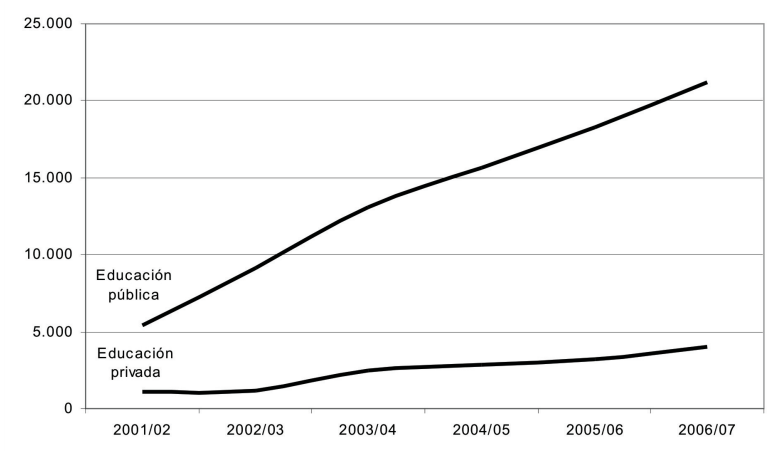

Fuente: Elaboración propia a partir de Ministerio de Educación (MEC).

\section{CONCLUSIÓN}

Creemos que este breve recorrido por la inmigración en la Comunidad Valenciana puede haber ilustrado cómo una región secularmente desarrollada por su situación geográfica y sus potencialidades económicas, sin olvidar la dualidad entre interior montano y litoral costero, ha sido, como el resto de la Península, emisora de emigrantes en algún momento de su historia, para pasar a ser, siguiendo también la 
tendencia casi general de España, un área receptora neta de inmigración nacional desde los años 60 del siglo XX y sobre todo de inmigración extra española en los albores del siglo XXI. Este último fenómeno, ha supuesto considerables beneficios para la sociedad y la economía valencianas que han visto repuntar su natalidad desde unos niveles preocupantemente bajos así como aumentar paralelamente, con la incorporación al sistema laboral de población joven foránea, el número de cotizaciones a la Seguridad Social en un contexto de población envejecida que no auguraba un buen futuro al sistema de pensiones públicas. Estas aportaciones de la inmigración no han recibido compensaciones por parte de la sociedad y la economía valencianas. Esto es así porque los trabajadores foráneos, a pesar de tener, en muchos casos, niveles de formación superiores a los de la población activa autóctona, han desempeñado trabajos poco cualificados. Pero lo que es más grave es que han visto como sus hijos e hijas si bien accedian a los servicios públicos de los que ya disfrutaban los residentes nacionales, eran, dentro de uno de los servicios sociales más importantes como es la Educación, progresivamente situados en una enseñanza pública devaluada y guetizada. De esta educación pública huían los estudiantes autóctonos buscando una enseñanza concertada y privada en gran medida vedada a la población extranjera. No obstante, hay que reconocer que algunas colonias como la inglesa o la noruega también promueven la autosegregación o segregación voluntaria fomentando la creación de colegios e institutos propios. Esta situación debe invitar a la reflexión de las autoridades competentes para evitar caer en dinámicas cuyos resultados se ha mostrado muy negativos en otros países occidentales que no han sabido integrar a un capital humano imprescindible para su funcionamiento social y económico (Valero Escandell, J.; 2002).

\section{BIBLIOGRAFÍA}

CONSELLERIA D'EDUCACIÓ (Generalitat Valenciana). CONSELLERIA DE SANITAT (Generalitat Valenciana).

CRUZ OROZCO, J. (1988): «Las áreas de montaña valencianas: crisis y reactivación». En Cuadernos deGeografía nº 44 págs. 183-202.

ESPARCIA PEREZ, J. (2002): "La creciente importancia de la inmigración en las zonas rurales de la Comunidad Valencianan. En Cuadernos de Geografía no 72 págs. 289-306.

GARCIA BARBANCHO, A. (1967): Las migraciones interiores españolas. Estudio cuantitativo desde1900. Instituto de Desarrollo Económico. Madrid, 75 págs.

GOZÁLVEZ PÉREZ, V. (1993): «La población valenciana durante la década 1981-1991». En Investigaciones geográficas n 11 págs. 131-152.

GOZÁLVEZ PÉREZ, V. (2004): "Evolución de la población valenciana durante la época emigratoria (1857-1960)». En Historia, clima y paisaje: estudios geográficos en memoria del profesor ANTONIO LÓPEZ GÓMEZ. Universitat de València. València, págs. 141-154. INSTITUTO NACIONAL DE ESTADÍSTICA (INE).

LÓPEZ PINO, C. (2004): «El mercado laboral de la población inmigrante en España, un estudio de cason. En Innovar (Revista de Ciencias Administrativas y Sociales en línea), n² 23 págs. 126-146.

MENAGES MENAGES, A. (2007): Els valencians d'Algèria (1830-1962). Memòria i patrimoni d'unacomunitat emigrada. Edicions del BuIlent. València, 256 págs.

MINISTERIO DE EDUCACIÓN Y CIENCIA (MEC).

MINISTERIO DE TRABAJO Y ASUNTOS SOCIALES (MTAS).

REVENGA ARRANZ, E. (1980): "La transición demográfica en España». En Revista española de Investigaciones sociológicas, nº 10 págs. 233241.

VALERO ESCANDELL, J. (2002): „Hacia una escuela multicultural: la presencia creciente de inmigrantes en el sistema educativo español». En Cuadernos de Geografía no 72 págs. 157-172. 
